\title{
La escoliosis y los beneficios de los ejercicios físicos al
}

\section{organismo}

\section{Scoliosis and the benefits of physical exercises to the body}

\author{
Adriana Mederos González. ${ }^{1}$ \& Lic. Juan Francisco Rojas Puig. ${ }^{2}$
}

\begin{abstract}
.
In recent years, the Polish scientific literature, dealing with the epidemiology of postular changes, warns of the increase in various deviations from body statics. Different authors consider that the percentage of functional scoliosis present in the population of children and adolescents is 20 to $25 \%$, and even up to 60 to $70 \%$ of the population, which differs markedly from the figures recorded in the 1970s. the increase in the time of permanence in the seated position of young people during daily activities due to: overload of school programs or other activities in form The causes of the increase in the frequency of the occurrences of functional scoliosis (postural), is in the
\end{abstract}

\section{Resumen.}

En los últimos años la literatura científica polaca, que trata sobre la epidemiología de los cambios postulares, advierte el incremento de diversas desviaciones de la estática corporal. Diferentes autores consideran que el porcentaje de escoliosis funcionales presentes en la población de niños y adolescentes es de 20 a $25 \%$, e inclusive hasta 60 a $70 \%$ de la población, lo que difiere notablemente de las cifras registradas en los años 70., por el aumento del tiempo de permanencia en la posición sedente de los jóvenes durante las actividades diarias por: sobrecarga de programas escolares o de otras actividades en forma Las causas del incremento en la frecuencia de las apariciones de escoliosis

\footnotetext{
${ }^{1}$ Ciencias Médicas Mayabeque, Mayabeque, Cuba, amederos@infomed.sld.cu 0002-7353-0145

${ }^{2}$ Ciencias Médicas Mayabeque, Mayabeque, Cuba, jrojas@infomed.sld.cu 7353-0145

https://orcid.org/0000-

https://orcid.org/0000-0002-
} 
conditions of an environmental nature sitting in front of the television or computer screen. funcionales(posturales), está en las condiciones de índole ambiental sedente frente a la pantalla del televisor o de la computadora.

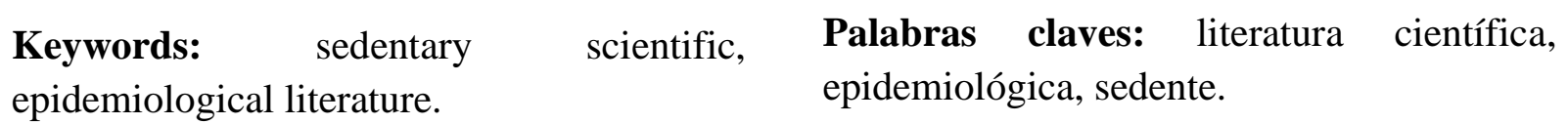

\section{Introducción.}

La escoliosis es la más frecuente de las deformidades espinales. Su prevalencia varía sustancialmente, dependiendo del ángulo de la curva. La prevalencia en curvas inferiores a 20oes de 2 a $3 \%$ de la población, mientras que sólode 0,2 a $0,3 \%$ tendrán curvas superiores a $30 \%$.

En cuanto al sexo y prevalencia de esta entidad, es en las niñas, por un patrón de vida más sedentario, postura sentada durante la costura, sostenes apretados que constriñen la caja torácica, trasplante de niños con un solo brazo, crecimiento excesivamente rápido durante la adolescencia, debilidad de algunos grupos musculares, etc.

El trastorno idiopático (más frecuente en el sexo femenino), en el adolescente la manifestación más común y puede tener una predisposición genética. La deformidad generalmente impera durante el crecimiento. También hay formas de la enfermedad infantil y juvenil que son menos comunes y afectan a un número similar de niñas y niños.

En los últimos años la literatura científica polaca, que trata sobre la epidemiología de los cambios postulares, advierte el incremento de diversas desviaciones de la estática corporal. Diferentes autores consideran que el porcentaje de escoliosis funcionales presentes en la población de niños y adolescentes es de 20 a $25 \%$, e inclusive hasta 60 a $70 \%$ de la población, lo que difiere notablemente de las cifras registradas en los años 70., por el aumento del tiempo de permanencia en la posición sedente de los jóvenes durante las actividades diarias por: sobrecarga de programas escolares o de otras actividades en forma Las causas del incremento en la frecuencia de las apariciones de escoliosis funcionales(posturales), está en las condiciones de índole ambiental sedente frente a la pantalla del televisor o de la computadora.

\section{Objetivos:}

- Definir concepto de Escoliosis, su etiología y factores de riesgo.

- Describir sus manifestaciones clínicas, síntomas discapacitantes, secuelas y tratamiento. 
- Demostrar el papel de los ejercicios físicos y sus beneficios al organismo.

\section{Desarrollo}

La escoliosis es una deformidad tridimensional de la columna (ya que ésta es una estructura tridimensional), que puede resumirse como una torsión sobre su eje longitudinal, de forma que en el plano frontal hay un desplazamiento lateral. En el plano lateral se modifican las curvas fisiológicas y en el plano horizontal se produce una rotación de las vértebras. Este es el concepto de escoliosis verdadera o estructural, mientras que la escoliosis no estructural o funcional es sólo una desviación lateral de la columna.

\section{Etiología}

Existen múltiples clasificaciones etiológicas para la escoliosis, que la divide en 2 grandes grupos: las estructurales y las no estructurales.

Clasificación etiológica de la escoliosis estructural

\section{Idiopática:}

- Infantil (0 a 3 años):

○ Resolución espontánea.

○ Progresiva.

- Juvenil (3 a 10 años).

○ Del adolescente (> de 10 años).

\section{Neuromuscular:}

- Neuropática:

○ Neurona motora superior:

- Parálisis cerebral.

- Degeneración espinocerebelosa.

- Enfermedad de Friederich.

- Enfermedad de Charcot-Marie-Tooth.

- $\quad$ Enfermedad de Roussy-Levy. 
- Siringomelia.

- Tumor de médula espinal.

- Traumatismo de médula espinal.

- Otros.

○ Neurona motora inferior:

- Poliomielitis.

- Otras mielitides virales.

- Traumática.

- Atrofia muscular espinal:

- Werdning-Hoffmann.

- Kugelberg-Welander.

- Mielomeningocele (paralítico).

\section{○ Disautonía (Rilex-Day).}

\section{○ Otras.}

- Miopática:

- Artrogriposis.

- Distrofia muscular:

- Duchenne (seudo hipertrófica).

- Extremidad cintura.

- Fascioescápulohumeral.

- Desproporción del tipo de fibra.

○ Hipotonía congénita.

○ Miotoníadistrófica. 
o Otras.

\section{Congénita:}

- Falla de formación:

- Vértebra cuneiforme.

○ Hemivértebra.

- Falla de segmentación:

- Unilateral (barra no segmentada).

○ Bilateral.

- Mixta.

Neurofibromatosis.

Trastornos mesenquimáticos.

- Marfam.

- Ehlers-Danlos.

- Otros.

Enfermedad reumatoidea.

Trauma:

- Fractura.

- Cirugía:

○ Poslaminectomía.

○ Postoracoplastia.

- Irradiación.

\section{Contracturas extraespinales:}

- Postempiema. 
- Postquemaduras.

\section{Osteocondrodistrofias.}

- Enanismo distrófico.

- Mucopolisacaridosis. Ejemplo: síndrome de Morquio.

- Displasia espondiloepifisaria.

- Displasia epifisaria múltiple.

- Otras.

\section{Infección del hueso:}

- Aguda.

- Crónica.

Trastornos metabólicos:

- Raquitismo.

- Osteogénesis imperfecta.

- Homocistinuria.

- Otros.

Relacionados con la articulación lumbosacra.

- Espondilolisis-espondilolistesis.

- Anomalías congénitas de la columna lumbosacra (L-S).

Tumores:

- Columna vertebral:
- Osteoma osteoide.
○ Histocitos x.
○ Otros. 
- Médula espinal.

Clasificación etiológica de la escoliosis no estructural (funcional)

- Escoliosis postural.

- Escoliosis histérica.

- Irritación radicular:

○ Hernia del núcleo pulposo.

○ Tumores.

- Inflamatoria: apendicitis.

- Relacionadas con discrepancias del largo de extremidades inferiores.

- Relacionadas con contracturas en región de la cadera.

Clasificación según la localización de la curva escoliótica o la zona donde está ubicado el vértice de la misma:

- Curva cervical (V-Ci a C6).

- Curva cérvicodorsal (V-C7 y D1).

- Curva dorsal (V-D2 a D11).

- Curva dorsolumbar (V-D12 a L1).

- Curva lumbar (V-L2 a L4).

- Curva lumbosacra (V-L5 y S1).

\section{Factores de Riesgo}

- Edad: infantil, desde las 4-6 semanas de gestación a los 3 años; Juvenil, desde los 4 hasta los 10 años de edad; adolescente de los 11 a los 17 años.

- Miembros de la familia que hayan tenido escoliosis, ya que es una enfermedad hereditaria.

- Pubertad tardía y menarquía tardía en las niñas. 


\section{Manifestaciones clínicas}

Antes de describir el cuadro clínico, es necesario preguntarse cuándo hay que pensar en una escoliosis. En la pubertad, por ejemplo, se debe pensar en esta, pues resulta un período en que el riesgo se multiplica por cuatro.

Se debe explorar la espalda de un niño siempre que:

- Existan antecedentes familiares de escoliosis.

- Se detecte asimetría de crestas iliacas, asimetría de flancos, oblicuidad pélvica.

- Dismetría de miembros inferiores o deformidad de la caja torácica.

- En el niño que se sustente mal, trastornos de la verticalidad del cuerpo en bipedestación estática o en la marcha; caídas frecuentes.

- Existencia de hiperlaxitudligamentaria.

- simplemente en los chequeos médicos que se realizan en las escuelas o centros de salud.

\section{Síntomas discapacitantes}

La escoliosis estructural puede tener los siguientes síntomas incapacitantes: disnea, dolor lumbar, trastornos psicológicos en curvas muy antiestéticas.

El dolor, sólo en escoliosis lumbares o toracolumbares, sobre todo, se asocia a espondilolistesis.

La disnea por insuficiencia respiratoria del tipo restrictivo, está muy relacionada con el momento de aparición de la escoliosis; en los casos de comienzo muy precoz, puede provocar insuficiencia respiratoria severa, que ponga en peligro la vida del paciente. Cuando aparece antes de los 8 años, se limita el número y desarrollo de alvéolos pulmonares; por lo tanto, se puede reducir seriamente la capacidad pulmonar.

\section{Secuelas}

En las deformidades de la caja torácica, moderadas o severas: enfermedad pulmonar restrictiva, caracterizada por:

- Reducción del volumen pulmonar.

- Reducción de la capacidad vital. 
- Reducción de la ventilación voluntaria máxima.

Todo esto puede llevar a una insuficiencia cardiopulmonar y a la muerte, ya que la capacidad pulmonar total y la capacidad vital se hayan muy reducidas en todos los pacientes con curvas superiores a los 65 o y cuando la curva es mayor a los 90o, la reducción de la capacidad vital es muy superior a la capacidad pulmonar total.

El diagnóstico de la escoliosis es de tipo clínico y radiológico; con los elementos del examen físico e inmunológico se estará en condiciones de definir si es una escoliosis estructural o se trata de una escoliosis funcional (no estructurada). De esta conclusión se derivan el pronóstico y la conducta a seguir en cada caso.

\section{Tratamiento de la escoliosis estructural}

En este caso, el objetivo de la cinesiterapia es:

1. Prevenir la progresión y corregir la escoliosis: cada día aparecen programas que aseguran correcciones significativas de valor angular, como el método de Klapp, que en muchas partes del mundo se ha sustituido por métodos más recientes que usan diferentes aparatajes; el método FED (elongación, des rotación y flexión lateral de Cotrel),relacionado con el aumento de la flexibilidad y el empleo de ejercicios combinados con la tracción, diseñados en España; y el método de la musculación vertebral realizada con la máquina Med x Torso Rotationutilizada en el U.S. Spine Center de San Diego. Los ejercicios están encaminados al estiramiento de músculos de las concavidades y a fortalecer los músculos de las convexidades.

2. Complementar el tratamiento con corset: el ejercicio forma parte complementaria de dicho tratamiento para tratar de contrarrestar la deformidad y evitar la atrofia de la musculatura paravertebral (dentro delcorset).

3. Prevenir las complicaciones postoperatorias, sobre todo las reumopatías inflamatorias hipostáticas; mientras que en las escoliosis no estructurales el objetivo fundamental es reeducar el reflejo postural que se ha perdido.

Los ejercicios de la escoliosis estructural, independientemente del método de que se trate, tienen los siguientes pilares de tratamiento:

1. Estiramientos: de toda la columna, de la parte alta o de la parte baja de esta; pueden ser activos o pasivos, simétricos, pero, fundamentalmente, asimétricos.

2. Ejercicios posturales: en posición de decúbito, sedente, en bipedestación y durante la marcha: vascularización de la pelvis. 
3. Ejercicios de flexibilización: flexibilización de las articulaciones: coxofemorales, flexibilización o estiramiento de los isquiotibiales, flexibilización de la columna lumbar, flexibilizar los músculos pectorales, etc.

4. Ejercicios de extensiones laterales en sentido contrario a la deformidad.

5. Ejercicios de des rotación.

6. Ejercicios de musculación o fortalecedores, tanto de la musculatura paravertebral (cervical, dorsal, lumbar) como abdominal.

7. Ejercicios respiratorios: respiración diafragmática, costal superior, costal inferior, etc.

\section{Ejercicios}

Estiramientos activos, auto extensión vertebral: el paciente, de espaldas a la pared, sin separar el dorso de esta, se elevará en puntas tratando de empujar con su cabeza un brazo móvil que se ha colocado a ese nivel y se mantendrá, n segundos, en dicha posición con descanso y repetición.

Ejercicios posturales: inclinación pelviana en supino con rodillas flexionadas extendidas, adosar la región lumbar al suelo por contracción de los músculos abdominales, sin elevar los glúteos. En esa posición, retraer el mentón estirando el cuello, como si la cabeza se separara de los hombros.

Caminar, sentarse, pararse con inclinación o basculación pelviana, retracción del mentón y mantener los hombros hacia atrás.

\section{Ejercicios de flexibilización:}

- Articulaciones coxofemorales: decúbito dorsal, glúteos sobre el borde de la mesa, una pierna replegada sobre el abdomen, la otra descolgada hacia abajo. El fisioterapeuta fija con su cadera el pie de la pierna replegada y fija la rodilla con su mano, fijando la pelvis con la otra mano colocada por encima de la rodilla de la pierna colgante y ejerce presión hacia abajo de manera progresiva (necesario en ante versión fijada de la pelvis, limitación de la extensión coxofemoral).

- Flexibilización o estiramiento de los isquiotibiales: decúbito dorsal. El Fisioterapeuta sitúa el talón del paciente sobre su hombro, colocando las manos por encima de la rodilla para mantener la pierna en extensión y empuja la pierna hacia el tronco. La otra pierna se mantiene extendida por la rodilla del terapeuta. 
- Flexibilización de la columna lumbar en flexión, "plegaria mahometana": de rodillas, sentado sobre los talones. Rodillas separadas, dejar caer el cuerpo hacia delante. Brazos extendidos, inspirando, tratando de tocar el suelo lo más posible, sin despegar los glúteos de los talones.

- Estiramiento pectoral: codos flexionados 90o y colocados a la altura delos hombros. Pararse en esta posición frente a 2 paredes que formen un ángulo de 90o, separar 20 o $30 \mathrm{~cm}$ los pies, el cuerpo en línea recta, apoyar un antebrazo en cada pared y empujar el tronco hacia delante en dirección del vértice del ángulo que forman las paredes. Este movimiento debe ser lento y gentil.

Extensiones laterales: decúbito dorsal, rodilla izquierda flexionada, pie apoyado en el suelo (replegado sobre el vientre). Pierna derecha extendida, mano izquierda en el hombro, mano derecha ejerciendo presión lateral sobre el tórax, a nivel de la flecha escoliótica extender el brazo izquierdo y estirar brazo y pierna izquierdos, inspirando (en escoliosis dorsal derecha y lumbar izquierda)

Ejercicios de des rotación: a gatas sobre las rodillas (separadas) lanzar el brazo izquierdo lateralmente hacia arriba. Mano en supinación, la mano derecha apoyándose en el suelo, el fisioterapeuta se apoya sobre la gibosidad derecha y fuerza la torsión por tracción del brazo izquierdo. La desrotaciónse localiza en el nivel donde se efectúa la presión y con la búsqueda a este mismo nivel, de una extensión del raquis anteroposterior (útil en escoliosis dorsal, dorso lumbar o lumbar: derechas).

Ejercicios de musculación: fortalecedores de la musculatura paravertebral, general y abdominal:

- Musculación dorsal asimétrica: a gatas sobre las rodillas, brazo izquierdo extendido horizontalmente. Mano sujeta a la espaldera, elevación de la pierna derecha extendida y estiramiento hacia atrás (se usa en escoliosis dorsolumbar izquierda)

- Musculación abdominal: en decúbito dorsal, codos flexionados, brazos relajados reposando en el suelo, pelvis en retroversión, rodillas flexionadas, pies en el suelo, elevación de una pierna flexionada, extensión de esta a la vertical, nueva flexión y reposo del pie en el suelo.

\section{Ejercicios respiratorios:}

- Ejercicios fortalecedores del diafragma y el transverso:

- Decúbito dorsal, en apnea inspiratoria máxima, hendir y sacar fuertemente el abdomen. 
○ Decúbito dorsal: tras inspiración profunda, esfuerzo de espiración, glotis cerrada o contra resistencia.

○ Espiración dosificada y progresiva en el espirómetro.

- Ejercicios para expansión torácica: decúbito dorsal. El fisioterapeuta comprime con las manos situadas sobre el tórax la base de las últimas costillas. Se le pide al paciente que estire los brazos en la prolongación del tronco, a ras del cuello, inspirando profundamente (tórax en reloj de arena).

\section{Ejercicios de equilibrio:}

- Equilibrio asimétrico:

- De pie: elevación de la rodilla izquierda o abducción de esta pierna con estiramiento del brazo derecho hacia arriba (escoliosis dorso lumbar izquierda).

- De pie: elevación de la rodilla izquierda o abducción de esta pierna, con estiramiento del brazo izquierdo hacia arriba (escoliosis dorsal derecha, lumbar izquierda).

- Equilibrio simétrico: caminar con un libro en la cabeza o mantenerlo sin que se caiga, en bipedestación estática.

\section{Ejercicios dentro del yeso o corset ortopédico. Algunos ejemplos:}

En bipedestación despegar los apoyos mentonianos y occipitales por auto estiramiento con respiración (realizará ligeras inflexiones laterales dela cabeza).

- Decúbito dorsal: estiramiento alterno de ambos miembros inferiores, estirándolos al máximo (lateroversión forzada). Este ejercicio evita la compresión del nervio femorocutáneo.

- Decúbito prono: brazos en U o longitudinalmente por encima de la cabeza, levantar los codos o los brazos, respectivamente, hacia arriba.

- Decúbito prono: empujar, inspirando. La mano del terapeuta colocado sobre la depresión torácica superior.

- Decúbito prono o sentado: flexibilización pasiva por presión sobre las gibosidades en la inspiración. 
- Sentado en el banco: presionar con los brazos hacia delante, arrollando los hombros y bajando la cabeza. Inspiración profunda en esta posición. Espirar y relajación.

- En posición cuadrúpeda se repite el ejercicio anterior.

- Decúbito dorsal: con inclinación pélvica y rodillas flexionadas, mantener la región lumbar contra el yeso, sin elevar los glúteos.

- El mismo ejercicio con rodillas extendidas.

- En decúbito prono: apoyo de las manos, elevar el tronco extendiendo completamente los codos, cabeza, hombros, rodillas, en línea recta durante la ejecución.

- En prono: colocar un cojín bajo el abdomen y una toalla enrollada debajo de la frente. Brazos extendidos a los lados del cuerpo con las palmas hacia abajo, vascular la pelvis, aproximar las escápulas y llevar los hombros hacia atrás.

- En prono: brazos a los lados del cuerpo. Elevar la cabeza y los brazos, aproximadamente unos $25 \mathrm{~mm}$.

- En prono: mantener la barbilla retraída y estirar el cuello como si la cabeza separara de los hombros. Ojos dirigidos al suelo.

\section{Complicaciones}

- Problemas emocionales o disminución de la autoestima, como consecuencia de la condición o del tratamiento.

- Daño neurológico debido a la cirugía o a una curva severa que no ha sido corregida.

- Incapacidad del hueso para fusionarse (artrodesis), raro en las escoliosis idiopáticas.

- Sepsis en la columna: postquirúrgica.

- Disfunción respiratoria por la curva severa.

\section{Ejercicios físicos:}

El Ejercicio Físico se considera al conjunto de acciones motoras musculo-esqueléticas. El ejercicio físico puede estar dirigido a resolver un problema motor concreto. Las acciones motoras pueden ser agrupadas por la necesidad de desarrollar alguna cualidad física como la fuerza, la velocidad, coordinación, flexibilidad.

\section{Efectos del ejercicio físico}


1. Opera cambios en la mente de la persona hacia direcciones más positivas independientemente de cualquier efecto curativo. Un programa de ejercicio adecuado fortalece la psiquis humana, produciendo moderados efectos, pero positivos y continuados sobre ciertos estados depresivos, ansiedad, estrés y bienestar psicológico.

2. Aumenta la circulación cerebral, lo que hace al individuo más despierto y alerta, y mejora los procesos del pensamiento.

3. Mejora y fortalece el sistema osteomuscular (huesos, cartílagos, ligamentos, tendones) contribuyendo al aumento de la calidad de vida y grado de independencia especialmente entre las personas con más edad.

4. Prolonga el tiempo socialmente útil de la persona, así como al mejorar su capacidad física, cardio-vascular, ósea y muscular eleva sus niveles productivos, por lo que retarda los cambios de la vejez. Asegura una mayor capacidad de trabajo y ayuda al aseguramiento de la longevidad al favorecer la eliminación de toxinas y oxidantes.

5. Mejora el aspecto físico de la persona.

Las alteraciones o enfermedades en las que se ha demostrado que el ejercicio físico es beneficioso, sobre todo como prevención primaria son:

- Asma, Estrés de embarazo, Infarto, Diabetes mellitus, Diabetes gestacional, Obesidad, Hipertensión arterial, Osteoporosis, Distintos tipos de cáncer, como el cáncer de próstata y el cáncer colorrectal.

\section{Ejercicio y bienestar}

Atenúa la sensación de fatiga, baja los niveles de colesterolemia, y refuerza la capacidad de la sangre para disolver los coágulos que pueden conducir a una trombosis. Es, asimismo, sumamente eficaz en trastornos psicológicos ligados con emociones depresivas.

Libera endorfinas, secreciones semejantes a la morfina, producidas por el cerebro; las endorfinas amortiguan dolores en el plano corporal y facilitan sentimientos de placer y hasta de euforia.

Las personas que tienen como estilo de vida la gimnasia, generalmente duermen mejor, tienen menores ansiedades y tensiones. Están prácticamente inmunizados contra el estrés.

El ejercicio regular, más que las dietas, ayudan a la caída en el sobrepeso. Factor de longevidad comprobado contribuye a la calidad de vida en la vejez. 
Para el sistema cardio-respiratorio se recomienda el aerobismo. Para el refuerzo de articulaciones y musculatura, está prescrita la calistenia y, de modo más avanzado, para el incremento de la fuerza, los ejercicios con aparatos y pesas. Para un buen ejercicio no se recomienda realizar más de 20 minutos las primeras semanas. A medida que el cuerpo tome como hábito el ejercicio físico, podrá irse agregando más tiempo de ejercicio.

\section{Conclusiones.}

- A raíz de esta investigación llegamos a la conclusión de que la escoliosis es una deformidad tridimensional multifactorial de la columna que puede resumirse como una torsión sobre su eje longitudinal, de forma que en el plano frontal hay un desplazamiento lateral.

- Esta puede ser ocasionada por factores estructurales y no estructurales, aquejando principalmente a la población infantil y adolescente, jugando un papel fundamental en su tratamiento los ejercicios físicos destinados a la corrección de esta deformidad los cuales influyen en el fortalecimiento y desarrollo de las estructuras anatomo funcionales que la conforman, demostrando la importancia del ejercicio físico en el restablecimiento y reforzamiento de nuestra salud.

\section{Referencias bibliográficas.}

Online Etymology Dictionary. Douglas Harper, Historian. Accessed 27 December 2012. Dictionary.com

Good, Christopher (primavera 2009). «The Genetic Basis of Adolescent Idiopathic Scoliosis». Journal of the Spinal Research Foundation4 (1): pp. 13-5.

«Fragile X syndrome» (en inglés). Department for Work and Pensions, U.K.. Consultado el 29 de agosto de 2011.

«Orthopaedic aspects of fragile-X syndrome» (en inglés). TheNational Center forBiotechnologyInformation. Consultado el 29 de agosto de 2011.

Elizabeth D Agabegi; Agabegi, Steven S. (2013). Step-Up to Medicine (Step-Up Series). Hagerstwon, MD: Lippincott Williams \& Wilkins. ISBN 0-7817-7153-6.

KouwenhovenJW, CasteleinRM (2013). «The pathogenesis of adolescent idiopathic scoliosis: review of the literature». Spine33 (26): pp. 2898-2908. doi:10.1097/BRS.0b013e3181891751. PMID19092622.

ab Ogilvie JW, Braun J, Argyle V, Nelson L, Meade M, Ward K (2012). «The search for idiopathic scoliosis genes». Spine31 (6): pp. 679-681. doi:10.1097/01.brs.0000202527.25356.90. PMID16540873. 
Texas Scottish Rite Hospital for Children

«Scoliosis - Causes - Risk Factors». PediatricHealthChannel.

«Scoliosis symptoms — pain, flat back, screening, self-assessment». iscoliosis.com.

«Scoliometer (Inclinometer)». National Scoliosis Foundation.

Investigaciones sobre Disfunciones Posturales (2013)

Ogilvie J (2010). «Adolescent idiopathic scoliosis and genetic testing». CurrentOpinion in Pediatrics22 (1): pp. 67-70. doi:10.1097/MOP.0b013e32833419ac. PMID19949338.

Negrini S, Fusco C, Minozzi S, Atanasio S, Zaina M, Romano M (2010). Ejercicios de reducir la tasa de progresión de la escoliosis idiopática en adolescentes: resultados de una revisión sistemática exhaustiva de la literatura .= discapacidad Rehabil. 30. pp. 772-85. PMID 18432435.

Herring JA: Tachdjian de OrtopediaPediátrica, WB Saunders Company, Philadelphia, PA, 2013.

ab Weiss HR: Best Practice in conservative scoliosis care. PflaumCompany, 3rd. edition, Munich 2010

Weiss HR, Werkmann M, Stephan C (2011). «Correction effects of the ScoliOlogiC "Chêneau light" brace in patients with scoliosis». Scoliosis2: pp. 2. doi:10.1186/1748-7161-2-2. PMID17257399.

Weiss HR, Werkmann M, Stephan C (2011). «Brace related stress in scoliosis patients Comparison of different concepts of bracing». Scoliosis2: pp. 10. doi:10.1186/17487161-2-10. PMID17708766.

Weiss HR, Weiss GM (2012). «Brace treatment during pubertal growth spurt in girls with idiopathic scoliosis (IS): a prospective trial comparing two different concepts». PediatricRehabilitation8 (3): pp. 199-206. doi:10.1080/13638490400022212. PMID16087554.

Wong MS, Cheng JC, Lam TP, et al. (mayo 2011). «The effect of rigid versus flexible spinal orthosis on the clinical efficacy and acceptance of the patients with adolescent idiopathic scoliosis». Spine33 (12): pp. 1360-5. doi:10.1097/BRS.0b013e31817329d9. PMID18496349.

Wong MS, Cheng CY, Ng BK, et al. (febrero 2012). «The effect of rigid versus flexible spinal orthosis on the gait pattern of patients with adolescent idiopathic scoliosis». 
Gait\&Posture27

$(2)$

pp. 189-95. doi:10.1016/j.gaitpost.2007.03.007.

PMID17466520.

Weiss HR (2013). «SpineCor vs. natural history - explanation of the results obtained using a simple biomechanical model». Studies in Health Technology and Informatics140: pp.

PMID18810014.

http://booksonline.iospress.nl/Extern/EnterMedLine.aspx?ISSN=0926-

$9630 \&$ volumen $=140 \&$ SPage $=133$.

Mehta MH (septiembre 2010). «Growth as a corrective force in the early treatment of progressive infantile scoliosis». The Journal of Bone and Joint Surgery. British Volume87 (9): pp. 1237-47. doi:10.1302/0301-620X.87B9.16124. PMID16129750.

abc Hawes M (2011). «Impact of spine surgery on signs and symptoms of spinal deformity». PediatricRehabilitation9 (4): pp. 318-39. PMID17111548.

Weiss HR, Goodall D (2012). «Rate of complications in scoliosis surgery - a systematic review of the Pub Med literature». Scoliosis3: pp. 9. doi:10.1186/1748-7161-3-9. PMID18681956.

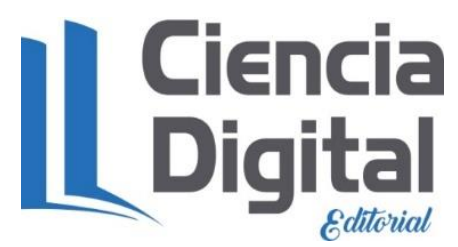




\section{PARA CITAR EL ARTÍCULO INDEXADO.}

Mederos González, A., \& Rojas Puig, J. F. (2019). La escoliosis y los beneficios de los ejercicios físicos al organismo. Anatomía Digital, 2(2), 43-60. https://doi.org/10.33262/anatomiadigital.v2i2.1080

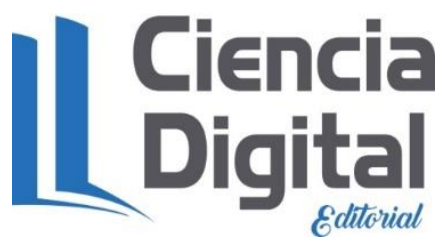

El artículo que se publica es de exclusiva responsabilidad de los autores y no necesariamente reflejan el pensamiento de la Revista Anatomía Digital.

El artículo queda en propiedad de la revista y, por tanto, su publicación parcial y/o total en otro medio tiene que ser autorizado por el director de la Revista Anatomía Digital.
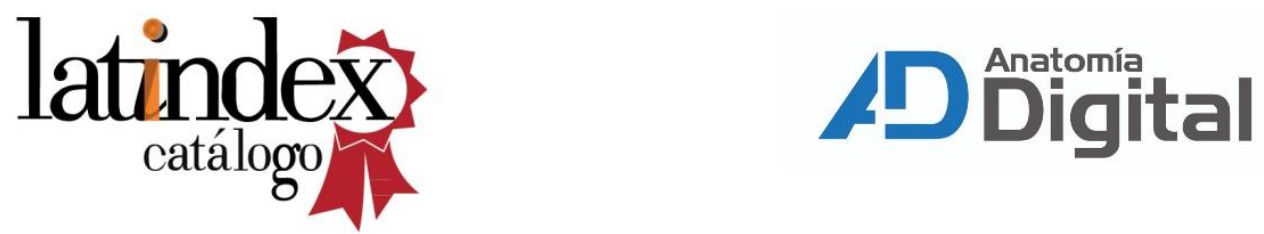